\title{
The Application of Traditional Architectural Decorative Art in Modern Architecture
}

\author{
Wei Xuefei \\ Yulin Normal University, Guangxi, China, 537000
}

\begin{abstract}
Keywords: architectural decorative art, structural veneer decoration, national culture, application
\end{abstract}

\begin{abstract}
In today's architectural art, it has become increasingly important to maintain and protect national traditions and regional cultures and to creatively inherit architectural art. Different historical backgrounds, cultural contexts and different geographical locations produce different buildings. Excellent architectural art is only placed in the foundation of their respective national cultures and they are widely recruited and borrowed for innovation. If you do not pay attention to history, do not pay attention to cultural connotation and do not pay attention to the protection of building entities, the resulting architectural decoration design will affect the integrity and coordination of the building. This paper briefly describes the traditional architectural art and the national characteristics and cultural implication of architectural decoration art and discusses the application of traditional architectural decorative art in modern architecture.
\end{abstract}

Decoration is one of the expressions of architectural art, aiming at beautifying architecture and architectural space. Chinese traditional architectural decoration is diverse in form, with a strong national and artistic nature, which greatly increases the artistic expression of architecture and is a beautiful miracle in the history of world architecture. In the architectural design, how to inherit this traditional art well, it is well used in modern architecture, combined with modern cultural connotation and spirit, forming a cultural tacit understanding, maintaining national tradition and regional culture,

\section{Traditional Architectural Art Analysis}

\subsection{Structural Decoration is divided into two}

One is the structural member of the wooden structure itself, such as the bucket arch, the beam column, the rafter, the rafter, etc., which belongs to the supporting member of the wooden structure building and has a good decorative effect by its own aesthetic sense and sequence sense. The other is based on the original structural members, modified, beautified, on the basis of the use of the addition of details, engraving, enriching the shape of the components, so that it plays a decorative effect. For example, Founder's beam head is used for modeling and it is engraved into Bawangquan; the Leigong column used in the spire structure is used to support the roof of the ridge, directly hanging under the treasure. The stigma below is usually made in the shape of a lotus flower; in the 
roof of the building, in order to increase the depth of the eaves, a spike is added to the front end of the tweezers. Usually, multiple curved surfaces are added and the shape is elegant; the column foundation is the column top stone of the underlying pillar. There are many styles of column foundations, such as the basin-type, drum-style vase-style, carved lotus pattern, dragon pattern, phoenix pattern, water pattern and even the whole animal stone sculpture. The bird's nest is placed on the top of the column. The members used to withstand the pressure of the upper part of the column are also usually engraved with engraving, or made into flowers, crepe and the like.

\subsection{Veneer Decoration}

The main function of the veneer is to waterproof and protect the structure. It is also the main embodiment of the building's appearance. Beautify the processing on the basis of the finish, add detail and greatly increase the artistic expression of the building. For example, if there is a drip tile in the position of the tile on the roof, there are various patterns: there are beasts, characters and lotus patterns; on the ends of the ridges there are tails, kisses, squids; There are white ash, gray plastic, painted, brick, stone, glazed flowers and other treatments.

\subsection{Decoration Decoration}

The decoration includes wooden doors and windows, partitions, flower covers and railings. The style and shape of the door are varied and the decoration on the door is also various. For example, the wooden lattice of various patterns used for the leaked part of the door, the wood carving of the solid wood board, etc.; the cover is a kind of indoor partition, which separates the space in the senses and continues ${ }^{[1]}$. Chinese traditional decoration is shown in Figure 1.

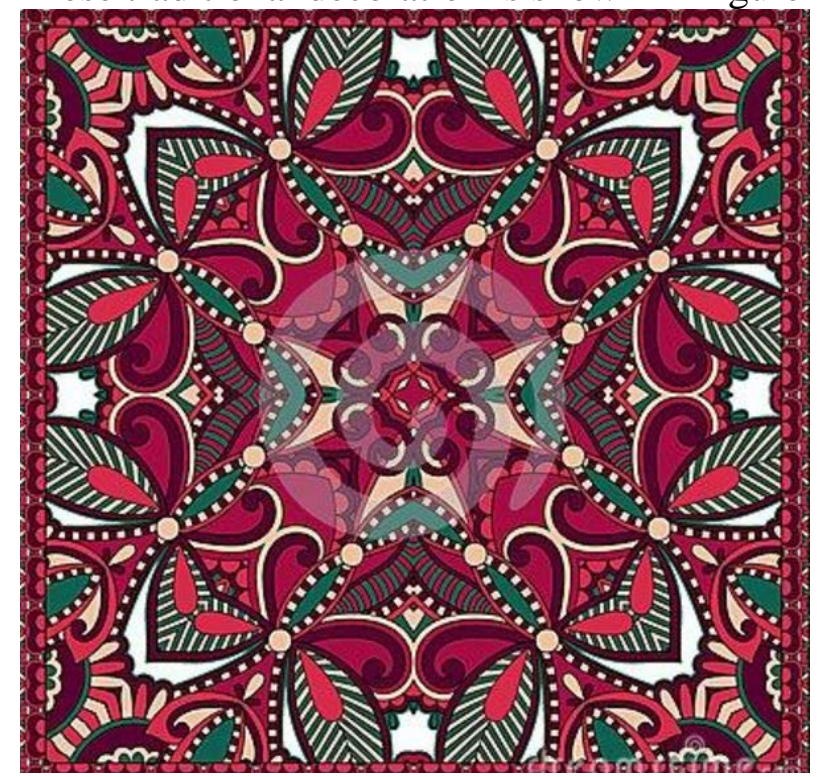

Figure 1 Chinese traditional decoration

\section{National Characteristics and Cultural Implication of Architectural Decorative Art}

The building is the main body and the decoration is the carrier. When the decoration is attached to the building, it shows the characteristics of the architectural culture. From the content and form of decoration, it can reflect the architectural culture characteristics of different ethnic groups and regions. In traditional folk architecture, the expression of ethics, folklore, culture and religious consciousness is often seen, as is the case with blue-sand roofs that are close to dark black. Qinghai 
is a folk custom and conceptual feature. Black represents the water in the ancient Five Elements. According to the principle of the five elements, the water is a fire, which means avoiding fire. Therefore, the use of green and black in the southern Chinese residential areas. The decorative patterns in the dwellings are more consistent with the real life wishes, using Fushoulu, plum, bamboo, chrysanthemum, pine and other forms. The cultural tradition of self-contained system stipulates a social concept, ideology and cultural form. In a country, architectural decoration forms a certain form under the influence of cultural traditions in a certain period, reflecting certain architectural art characteristics. With the continuation of culture, architectural decoration will also change and develop. In the practice of architectural decoration engineering, in the face of different decorative content, different geographical and human environments, different functional needs, different styles and cultural connotations of engineering projects, designers must reflect the artistic characteristics and creative personality, Exploring and researching the style of the main decorative content, the external factors of the style (ethnic characteristics, social system, lifestyle, cultural trends, technological development, customs, religious beliefs, climate products, geographical location) and style The intrinsic factors (individual or group creation ideas, including the creators' professional quality and artistic quality) are combined to give the content of the designed decoration a visual pleasure and cultural connotation, so that the high-tech, high-tempo life in modern society People are psychologically and spiritually balanced. They embody the various styles of artistic characteristics and creative personality into the specific projects designed, using material technology and architectural aesthetics to create functional, comfortable and beautiful. Excellent architectural decoration that meets people's material and spiritual needs ${ }^{[2]}$.

\section{Application Analysis of Traditional Architectural Art Deco in Modern Architecture}

\subsection{Built Entirely in Accordance with Traditional Architectural Forms}

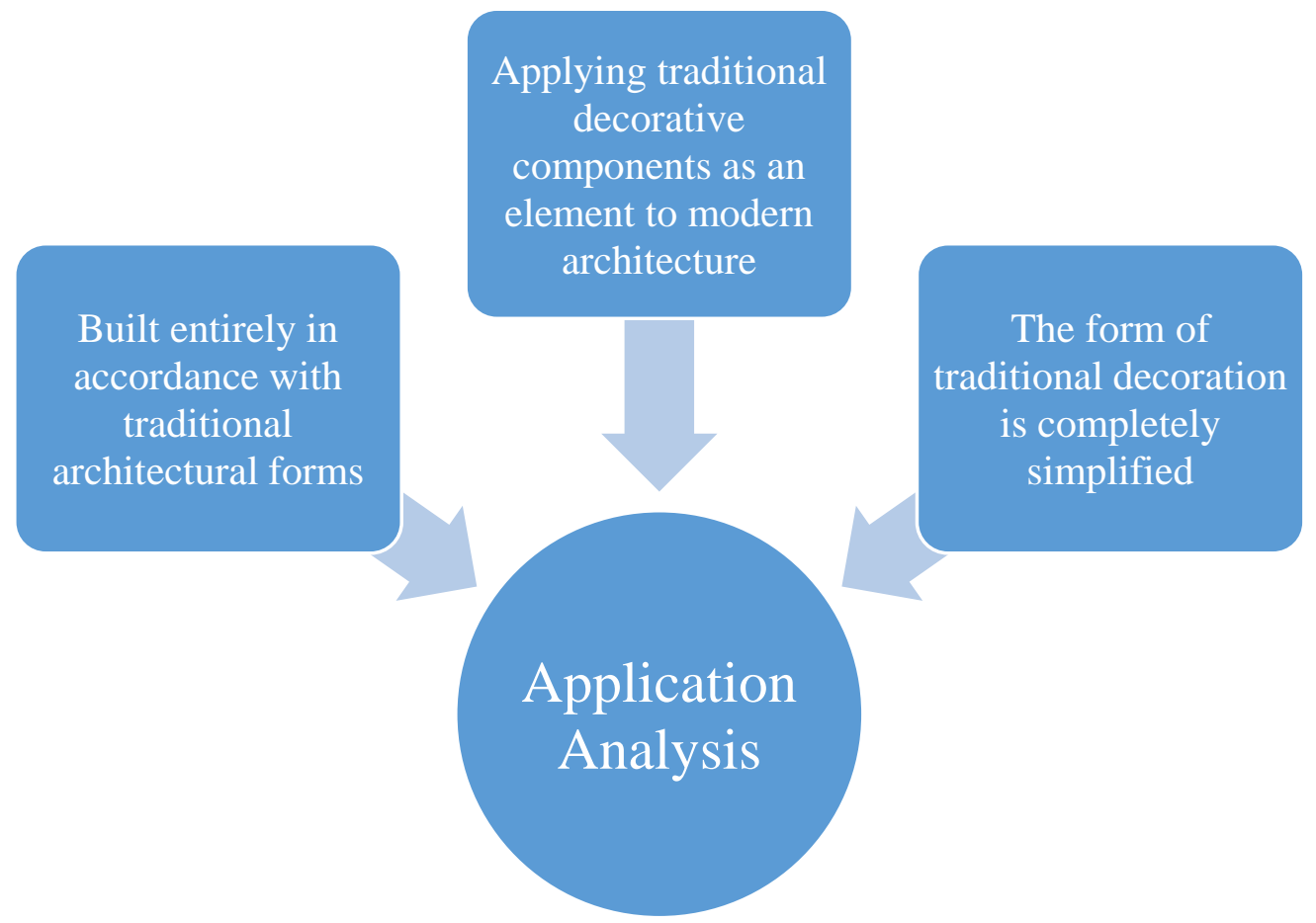

Figure 2 Several ways to apply traditional architectural art in modern architecture

Such as the traditional Chinese garden in Gyeonggi-do, South Korea - Yuehua Court, is such a 
project. The buildings are all in the form of traditional wood structures. Among them, the bow is even more elaborate. At the top of the bow of the bow, the three sides are open and the flying hood is decorated with hollow flowers and birds. The front hall is separated from the middle hall by a floor cover and the middle is an octagonal door hole. Both the central hall and the back hall are built on the top of the mountain ${ }^{[3]}$. The middle hall is made up of beautiful women on both sides and the back is hanged; the back hall is closed with window sashes, the upper part is the pane of the brocade, the lower part is the solid skirt and the brocade wood line is added. The entire building is exquisitely small, dignified and gorgeous, full of rich classical beauty. There are several ways to apply traditional architectural art in modern architecture, as shown in Figure 2.

\subsection{Applying Traditional Decorative Components as an Element to Modern Architecture}

In some buildings, both new architectural styles and Chinese-style charms are often used, often with traditional Chinese elements in the decoration ${ }^{[4]}$. For example, if a traditional window is built on a solid wall, it not only enriches the facade of the wall, but also penetrates the space and adds Chinese elements. For example, the traditional form of the partition is applied to the separation of different spaces. The use of exquisite classical lattices, which are separated and continuous, not only beautifies the space, but also allows two different spaces to be merged, so that you have me, I have you; there is also a practice of using some traditional components. As a special ornament. These components are not necessarily related to the new building. It can be said that they are used as a national symbol in places where they are needed, such as the flower door, the door nail, the paving head and so on. Practice shows that traditional architectural decoration can still play its role in modern architecture. Traditional decorations are also being updated and developed in constant use, gaining new vitality.

\subsection{The Form of Traditional Decoration is Completely Simplified}

For example, many modern Chinese-style residential buildings are now on pure white walls, with gray I-beam instead of blue bricks. From the fundamental changes in form and material, only people feel the Chinese style of residence from the tone and pattern. The exterior decoration of the whole building has both a modern architectural sense and the dual charm of Chinese traditional artisans. There is also a long vertical window hole on the real wall, which reflects the Chinese style through the bamboo strips that extend from the opposite side. artistic conception ${ }^{[5]}$.

\section{Conclusion}

With the development of economic construction, the art of architecture is changing with each passing day. More and more Chinese decorative elements have been applied to architectural design. In different periods, people have different requirements for space and their functionality, aesthetics and values have undergone tremendous changes. Therefore, it is necessary to analyze the feasibility of the space environment model in which the traditional and modern coexistence, the new and the old are unified. The way to continue the Chinese architectural art deco is to first understand the individual functions, characteristics and causes of decoration. Only in this way can we better master the artistic soul and use it in modern architecture.

\section{References}

[1] Mojtaba Parsaee,Parinaz Motealleh,Mohamad Parva. An investigation into the former consulate of Britain as one of the first samples of modern architecture in Iran[J]. HBRC Journal,2015.

[2]Fobel, C.,Grewal, G.,Stacey, D.. A scalable, serially-equivalent, high-quality parallel placement methodology 
suitable for modern multicore and GPU architectures [P]. Field Programmable Logic and Applications (FPL), 2014 24th International Conference on, 2014.

[3]Xin Liu,Chungjin Hu,Yang Li,Lina Jia. The Advanced Data Service Architecture for Modern Enterprise Information System [P]. Information Science and Applications (ICISA), 2014 International Conference on, 2014.

[4]Jozwiak, Lech. Automatic architecture exploration of massively parallel MPSoCs for modern cyber-physical systems [P]. Design and Diagnostics of Electronic Circuits \&amp; Systems, 17th International Symposium on, 2014.

[5]Yang You,Song, S.L.,Haohuan Fu,Marquez, A.,Dehnavi, M.M.,Barker, K.,Cameron, K.W.,Rand les, A.P.,Guangwen Yang. MIC-SVM: Designing a Highly Efficient Support Vector Machine for Advanced Modern Multi-core and Many-Core Architectures [P]. Parallel and Distributed Processing Symposium, 2014 IEEE 28th International, 2014. 